\title{
3D AutoSysLab Prototype - A Social, Immersive and Mixed Reality Approach for Collaborative Learning Environments
}

\author{
http://dx.doi.org/10.3991/ijep.v2i2.2083 \\ F. M. Schaf ${ }^{1}$, S. Paladini ${ }^{2}$ and C.E. Pereira ${ }^{2}$ \\ ${ }^{1}$ Universidade Federal de Santa Maria, Santa Maria, RS, Brazil \\ 2 Universidade Federal do Rio Grande do Sul, Porto Alegre, RS, Brazil
}

\begin{abstract}
Recent evolutions of social networks, virtual environments, Web technologies and $3 D$ virtual worlds motivate the adoption of new technologies in education, opening successive innovative possibilities. These technologies (or tools) can be employed in distance education scenarios, or can also enhance traditional learning-teaching (blended or hybrid learning scenario). It is known and a wide advocated issue that laboratory practice is essential to technical education, foremost in engineering. In order to develop a feasible implementation to this research area, a prototype was developed, called 3DAutoSysLab, in which a metaverse is used as social collaborative interface, experiments (real or simulated) are linked to virtual objects, learning objects are displayed as interactive medias, and guiding/feedback are supported via an autonomous tutoring system based on user's interaction data mining. This prototype is under test, but preliminary applied results indicate great acceptance and increase of motivation of students.
\end{abstract}

Index Terms-Engineering education, learning systems, collaborative tools, educational technology.

\section{INTRODUCTION}

The known advances in technology in many fields have broadened the possibilities of teaching and learning techniques and/or methodologies. Most prominent of this methodologies is blended or hybrid learning with help of virtual learning environments. This way, simple explanatory teaching and learning can be complemented and/or enhanced with collaborative and rich media learning objects.

Although for itself virtual learning environments (VLEs) offer the display and organization of didactic materials, the experimentation or laboratory practice is the key to shorten the gap between theory and the real world practice. For automation systems, training and teaching are crucial to link didactic lessons to industrial facilities real applications, i.e., offer experimentation. Nowadays, engineering education is a key aspect for most countries since it has been recognized that skilled engineers are one of the main components for the development of innovative products and services, as well as for the optimization of production processes to ensure high productivity and quality.

There is a trend among Higher Education Institutes to offer remote experiments as alternative to traditional laboratories where industrial or didactic equipments are expensive to maintain and restricted for security issues. This alternative is called the "Second Best of Being There" (SBBT) [1] and is an economic solution to expensive traditional (Hand-on) laboratories [2]. The appliance of remote experiments is not restricted to automation, they cover from: physics [3], aerospace applications [4], embedded communication systems [5], robot and other systems teleoperation [6, 7] to medicine [8]. These experiments utilize customized devices and software to make small-scale textbook-like experiments remotely available.

However, the availability of remote experiments is not a sufficient condition to ensure success in learning. Standalone remote experiments without connection to adequate learning material usually lead students to the trial and error strategy, which has a lower learning impact than originally expected [9] and decrease the added value of this alternative solution. Additionally, remote facilities are available 24-7, or anywhere, anytime, increasing the demand in the number of faculty members and tutors required to provide online guidance.

The use of VLEs in education opens up many possibilities to automate and adapt resources according to students' skills. Several advantages of the involved concepts can be integrated in a single environment. Among them are: collaboration, shared learning spaces, remote handling of experiments (haptic interfaces), mixed reality, immersive interfaces, social interactions, etc.

This work is organized as follows: Section 2 gives a background approach to virtual environments for learningteaching purposes, Section 3 presents the state of the art on related works, Section 4 describes the development of the prototype, in Section 5 conclusions are drawn, and finally Section 6 some future works are appointed.

\section{VIRTUAL ENVIRONMENTS FOR LEARNING-TEACHING}

Virtuality among several other utilities aims to close up dispersed or distributed users in a common locus. This "site" called commonly as environment is not physically real but virtual. Two well-known acronyms are directly related to virtual environments: the MUVEs (Multi-User Virtual Environments) and the MMORPG (Massively Multiplayer Online Role-Playing Game). While the first is a more generic term associated to all kinds of implementations of virtual worlds, the second is specifically employed in the entertainment field. There are several types 
of virtual environments that can be classified in areas of applications: work, learning, social and entertainment.

To achieve higher levels of human-human interactions, which are required to solve complex engineering problems, a strong support of collaboration and multiperspectivity is required. Most of the MUVEs allow and facilitate collaboration among users.

The following subsections describe and contextualize the terms: mixed reality, learning environments, social environments and immersive environments terms associated with Computer Supported Collaborative Learning (CSCL) [10], i.e., interfaces to learning-teaching.

\section{A. $\quad$ Mixed Reality}

As the name suggests, it is a mixture (blend) of realities, i.e., virtuality (computer simulated or not) combined with reality. The term was coined in 1994 by Paul Milgram [11]. Mixed reality interfaces can overlay graphics, video, and audio onto the real world. This allows the creation of shared workspaces that combine the advantages of both virtual environments and seamless integration (also collaboration) with the real environment [12].

Mixed reality can be applied in several levels: user interfaces (including virtual environments), experiments (didactic scenarios), user-to-user/system communication (interaction with other users or systems), etc. The information overlay is employed by remote collaborators to annotate the user's view, or may enhance face-to-face conversation producing shared interactive virtual models. In this way, mixed reality techniques can produce a shared sense of presence and reality [13]. Thus, mixed reality approaches are ideal for multi-user collaborative lab and work applications [12].

By analyzing the pros and cons of real vs. simulated experiments, one can see that in some sense they are complementary so that a combination of both possibilities seems interesting. The interchangeable components strategy (applied to automation systems) has been developed to allow the combination of both real and virtual components [14] supporting this way the definition of a variety of learning scenarios. In this strategy, automation system elements, i.e., controllers, plants, sensors, actuators are components that are combined in a variety of scenarios used to illustrate didactical situations (e.g. a real controller connected to a simulated plant - Hardware-in-the-Loop).

\section{B. Virtual Learning Environments (VLEs) Background}

Virtual Learning Environments or Learning/Course Management System (LMS/CMS) are the most common tools employed for organize digital media learning objects/materials available to distributed users on the Web. These tools are also related to Computer Based Training (CBT), Web Instruction (WI), Instruction Management System (IMS), Learning Objects Manager (LOM), and others.

Perhaps one of the most common free VLE implementation is MOODLE [15]. MOODLE is an open source project that is based on the social constructivism pedagogy. This pedagogic approach began with the popularization of the theories of Jean Piaget (Constructivist Theory of Knowing), Jerome Bruner (Cognitive Learning Theory) and most of all of Lev Vygotsky's. This social constructivism paradigms are based on the premises that: i. learning is an active and constructive process; ii. the learner is active during the whole process, building his own knowledge; iii. learners' subjective reality representations are previously linked to prior knowledge; iv. there are several teaching "techniques" but all involve collaboration and active interactions (e.g. debates, discussions, role playing games, etc.). Therefore, this pedagogic approach is well suited to VLEs, since it is suited for social interactions and learning [16].

Most VLE implementations aid teachers (instructors) with sufficient abstraction and intuitive graphical tools to create, host and link their didactic materials. They also provide user control and logging information that help to trace users' (students) interactions.

Concepts of learning are closely related to collaboration. On collaborative activities humans interact employing self-critiquing (reflection), inquiring and arguing skills. These skills propel the knowledge building. This is the very essence of the (social) constructivism pedagogy employed nowadays in virtual learning environments (or also virtual collaborative learning environments) and even in some special dedicated schools.

CSCL is a major method for bringing the benefits of collaborative and cooperative learning to users of distance learning via networked computers, such as courses offered via Internet. The purpose of CSCL is to scaffold or support students in learning together effectively. It supports the communication of ideas and information among learners, collaborative accessing of information and documents, and instructor and peer feedback on learning activities. Also supports and facilitates group processes and group dynamics in ways that are not achievable by face-to-face communication - such as having learners label aspects of their communication.

It is important to mention that the use of VLEs does not necessarily exclude the traditional education. A mixture of the traditional and the CSCL is also much more common and related in the literature as blended learning. This blending brings up advantages to the traditional education offering technology related aspects to learning that are nowadays undeniable and advocated even from the most backward educators.

\section{Social Environments}

While software may be designed to achieve closer social ties or specific deliverables, it is hard to support collaboration without also enabling relationships to form, and to support a social interaction without some kind of shared co-authored works.

Despite their lack of objective (at least they have open objectives), social networks allow connections and links between users promoting exchange of ideas, medias, and all kind of digital communication. This very essence can be captured aiming to educational purposes, giving the virtual environment a social "skin".

Softwares designed to social interaction are commonly called socialwares. The increasing audience of game or socialware implementations, like Second Life [17] and Active Worlds AWEDU [18], point out to a more gamelike solution applied to virtual environments with more interactive contents. Play ethic (methods) applied to work turn activities that employ computers a more comfortable experience. This is commonly referred to game-like interface. 


\section{Immersive Environments}

Immersion can be seen as the partial of full surrounding of user's senses (through use of images, sounds, etc.) into an environment (real or virtual). There are several techniques and equipments (normal displays, 3D displays, HMDs - Head Mounted Displays, CAVEs - Cave Automatic Virtual Environments, force-feedback interaction devices, etc.) to achieve different levels of immersion.

The most common immersion feeling is achieved by the use of avatars in tridimentional virtual environments, virtual worlds, or even metaverses (meta universes) giving the user the sense of beeing there (even if only virtually) [13]. Most of MMORPGs offer this interface. Immersed virtual users, avatars, navigate through the metaverse(s) walking and interacting with virtual objects.

Socialware implementations can have a 3D representation aiming to display more realistic (virtual) worlds to its users. An alternative association from social learning environments captive more attention from students while can focus on collaboration for education. Virtual environments that employ game-like interfaces in their design with purposes other than entertainment are referred as serious game.

\section{RELATED WORKS}

In order to pinpoint this work in the scientific community a wide range of state of the art research was conducted and the most prominent works of this study are presented here.

Virtual laboratories were the first implementations after simple Web tutorials to really add value to education, especially in the technical field where practice is necessary. Among the scientific community there is a wide range of results and VLabs available. The VCLab [5] is good example of virtual laboratories used for education that employ merely simulations to illustrate practical situations. Most of these simulations use common software tools like: MatLab [19], LabView [20] or similars to model and simulate the behavior of virtual experiments that mimic real practice or didactic scenarios.

The Automatic Control Telelab (ACT) proposed by [21] offers a remote lab facility, i.e., real experiments made accessible through the Web. This particular implementation supports not only controller parameterizations, but also MatLab Simulink models to describe and characterize the controller logic applied in the real experiment (plant). This interesting approach, called Software-in-theLoop, is very useful in the experiment configuration. Thus, students can design their own experiment controller in a much more flexible way.

The Solar Energy e-Learning Lab from [22] has an integrated learning system with several learning materials and "quizzes" to identify student understanding level. First, the student must pass several theory tests, so that the system grants remote experimentation access to a real solar energy plant facility.

Collaboratories [23] are a well known association of collaborative tools with remote laboratories (experiments). This solution brings up not only collaboration support but promotes that several students interact in a single experiment.

Another excellent example of flexible experiments configuration is the deriveSERVER system proposed by [24].
Unlike the others, this employs mixed reality techniques, using hyper-bonds [25] as bidirectional connectors, and is integrated in the VLE with collaborative and distributed learning methods.

An interesting approach employed in CSCEs (Computer Supported Collaborative Environments) is the use of CAVEs associated to mixed reality. In [26] such approach is described where CAVE canvases (projections) links collaborators in the same environment (CAVE). The common virtual workbench and the real workbench (via video projection) are available via Web and visible at an enlarged screen or beamed at canvases.

The project SLOODLE [27] presents a different approach that merges 3D world representation of Second Life with MOODLE to mirror Web-based classrooms with in-world learning spaces and interactive objects. Compared to other electronic tools for distance communication (Computer Mediated Communication - CMC), the metaverse representation improve the sense of being there (in a classroom), rather than of being a disembodied observer, like most $2 \mathrm{D}$ virtual environments. This representation employs state of art technologies that support collaboration, creativity and sharing over the Web.

In [28] a virtual campus is created with several didactic materials, simulations and also an immersive interface to remote laboratories in the social 3D World hosted by Second Life. This is a particularly close solution to the forthcoming proposed architecture.

In [3] a fully integrated experiment from MIT's iLabs [29] with immersive environment is created. Differently from [28] where the Second Life server infrastructure is used, the 3D Physics Lab used the Open Wonderland project [30], a free, open-source, Java based technology for virtual environment management.

In [8] results of the Project TOUCH (Telehealth Outreach for Unified Community Health) are reported, which involves remote practice applied to medicine. A remote surgery tool was developed to apply problem-based learning scenarios to medicine students.

Another related work in the medical learning is JDoc [31], which adds great motivational characteristics to instructional systems. JDoc is serious game implementation for medical training (disease diagnostics).

\section{PROTOTYPE DEVELOPMENT}

For the development of a prototype, a careful study of the state of the art of current technologies and concepts associated with virtual environments for teaching and learning was conducted. From this study a base architecture or framework for CSCEs was created and proposed in $[32,44]$. The architecture follows a model depicted in Figure 1.

The following subsections describe the prototype framework; the embedding of remote experiment interfaces into the 3D social metaverse; an automation systems simulated experiment on the metaverse; the extrapolation of this experiment using mixed reality techniques; the integration of interchangeable components in this experiment; the active collaboration within the metaverse; and a simple autonomous tutoring system approach. 


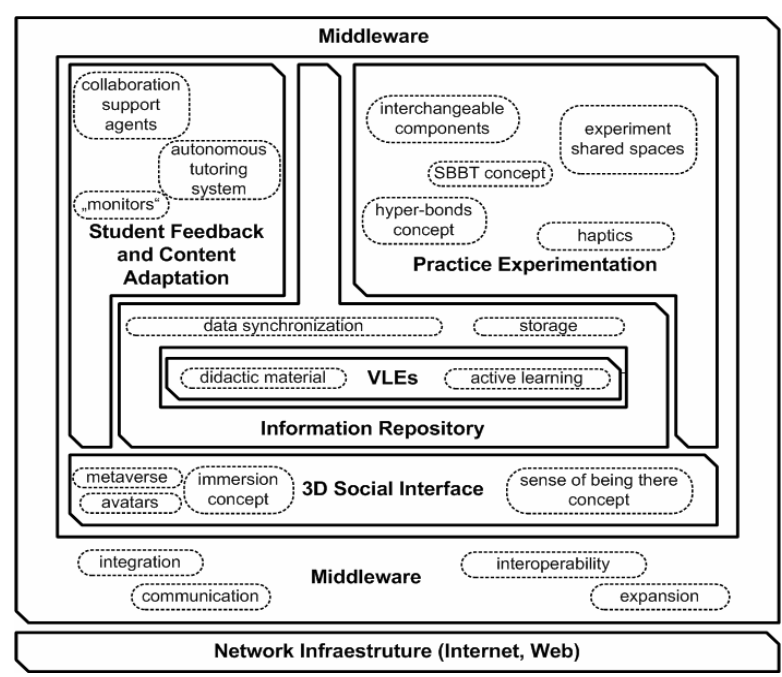

Figure 1. Concept modules diagram of the CSCE architecture

\section{A. Prototype Framework}

The hardware and software organization of the prototype was created to achieve:

- maximum usability - use of free and open-source software implementations/tools and also low cost hardware;

- adaptability and interoperability - use of software implementations that are standardized, with an active developing community and that employ communication interfaces that allows interoperability;

- distribution - hardware and software resources as well as operation/interaction are based on a distributed architecture of communication;

- friendly interface - require minimum users skills to navigate and interact in the virtual environment.

Using these principles, a base prototype for hosting metaverses and a simple virtual world with experiments related to control and automation engineering was created using the following software and hardware:

- MOODLE [15] - center of organization of didactic and learning material;

- Open Simulator [33] - as metaverse hosting server;

- Hippo OpenSim Viewer [34] - as user client interface to view, create, navigate and manage metaverses' contents;

- SLOODLE [35] modified package - for linking MOODLE to Open Simulator objects;

- JADE Framework [36] - to create agents responsible for data mining and simple autonomous tutoring feedback;

- MySQL [37] - as database (information repository) management system;

- LibOpenMetaverse [38] - to create autonomous tutors representations, i.e., avatars (bots). Also to link external resources (software and hardware) to the Open Simulator;

- Arduino Board [39] and MicroWebServer [40] - as simple hardware interface and controllers to link to several experiments;

- Several simple experiment hardware - LEDs, pushbuttons, motors, sensors, etc.

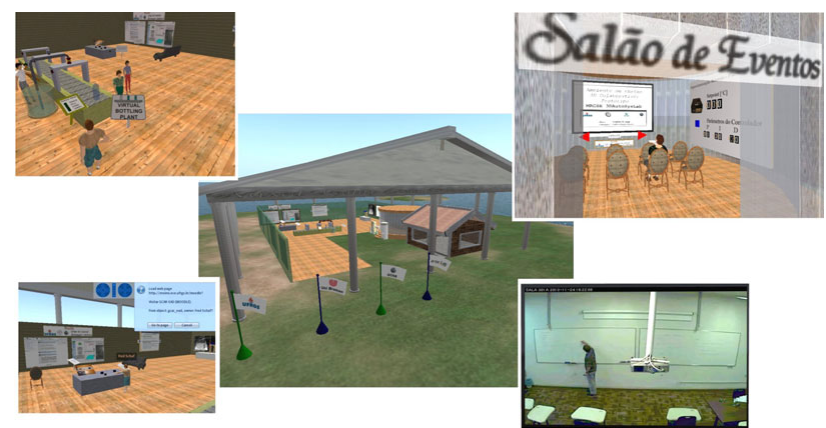

Figure 2. 3D AutoSysLab metaverse client interface

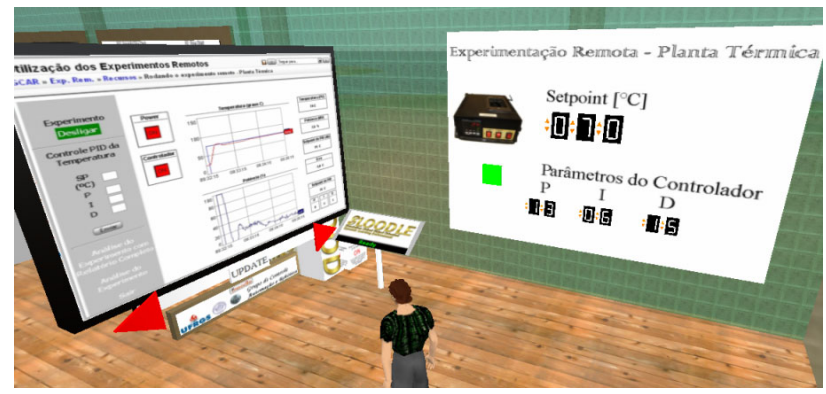

Figure 3. Immersive experiment interface

Of course several other hardware and software tools were developed since the mentioned softwares are not compatible for itself. This task is addressed to the middleware that provides a common ground for communication interface. Mostly of the communications are made by XML-RPC, but other simpler interfaces are also used (HTTP, PHP with MySQL).

The Figure 2 illustrates the 3D AutoSysLab metaverse snapshots. Some capabilities are evident, such as: immersive communication (interaction/collaboration) support, multimedia display (video/audio/pictures) support, virtual experimentation support, mixed reality experiments support, user-friendly interface, etc.

\section{B. Experiment Interface in 3D AutoSysLab Metaverse}

The first attempt to reshape the Web interface of the experiments available $[9,14]$ in the GCAR research group was done in to the remote experiment thermal plant. The goal of this experiment is to control the temperature of an electrical resistance by manipulation of the electrical current that flows through it. An industrial controller is used to control the current via PWM (Pulse-width Modulation) given PID control parameters. Figure 3 illustrates the proposed alternative experiment interface and a user conducting the experiment.

The experiment interface is embedded on the 3D social metaverse and the experiment can be controlled (run/stop and input of PID controller parameters) using simple inworld scripting (HTTP calls) to transmit to the experiment manager system its parameters.

\section{Virtual Bottling System Experiment}

In order to establish a didactic and easy to visualize virtual industrial plant, a metaobject, i.e., a virtual object, that represents a simple bottling system in the metaverse, was created. This virtual plant mimics a simple bottling system were the process of filling, corking and labeling the bottle is realized (see Figure 4). 


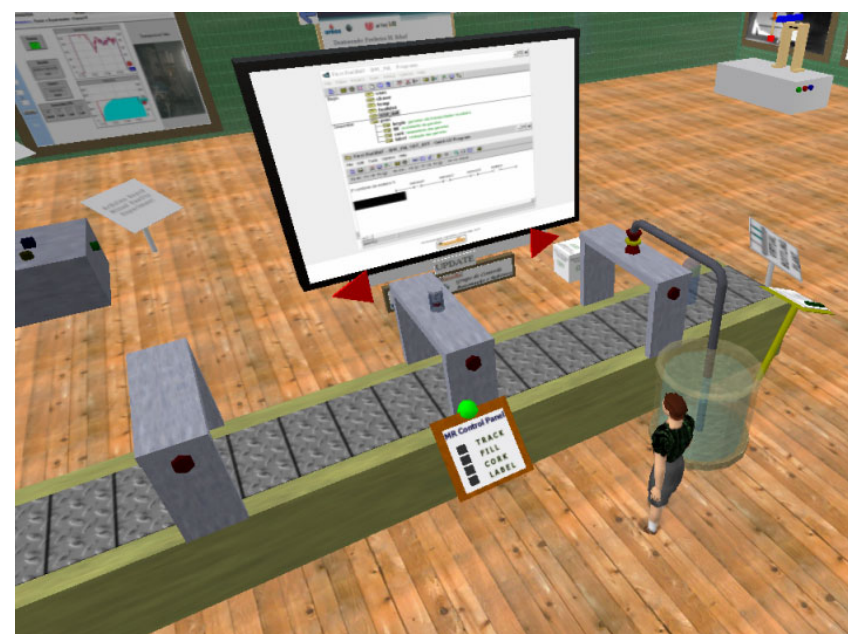

Figure 4. Learning material display and experiment interface

For the entire process four actuators are used: in the fill station a valve controlling the liquid flow; in the cork station a corker arm; in the label station the labeler arm, and additionally a conveyor belt motor to transport the bottle(s) through the stations. To automate the process only three sensors are used - each one to detect the presence of bottles at the stations.

This simple virtual experiment has several applications and can be used to illustrate several scenarios from automation system concepts to controller programming languages. Traditionally, students test their IEC 61131-3 compliant programs to automate the bottling system.

In order to supply learning material associated with the experiment the SLOODLE package was adapted (originally directed only to Second Life) to link previously developed didactic material of IEC 61131-3 programming languages hosted in MOODLE (see Figure 4).

\section{Mixed Reality Scenarios}

With the virtual bottling plant, a Hardware-in-the-Loop scenario was also developed using the Duemilanueve Arduino Board as controller or as real control panel interface [44].

Figure 5 displays the mixed-reality bottling system plant, which is used in several lectures, such as (i) for teaching PLC programming using with the IEC 61131-3 standardized programming languages for automation and (ii) in the development of micro-controller based sensor/actuator interfaces.

\section{E. Bottling System Interchangeable Components}

To propose the interchangeable components strategy [14], a simple real bottling system plant was created, using simple hardware (motor, sensors and mechanical parts) and the ARToolKit [41] to create the bottle in augmented reality, since the bottle transport and filling is a challenge in this experiment. This plant is depicted in the Figure 6.

To interface the plant with the computer (also OpenSim) a Micro Web Server (MWS) was used. The MWS controls a relay board by the use of CGI (Common Gateway Interface) over TCP/IP, i.e., can be accessed by any browser.

The developed simple real bottling system plant was integrated in to the Open Simulator by the creation of a simple script that execute HTTP calls, i.e., CGI running

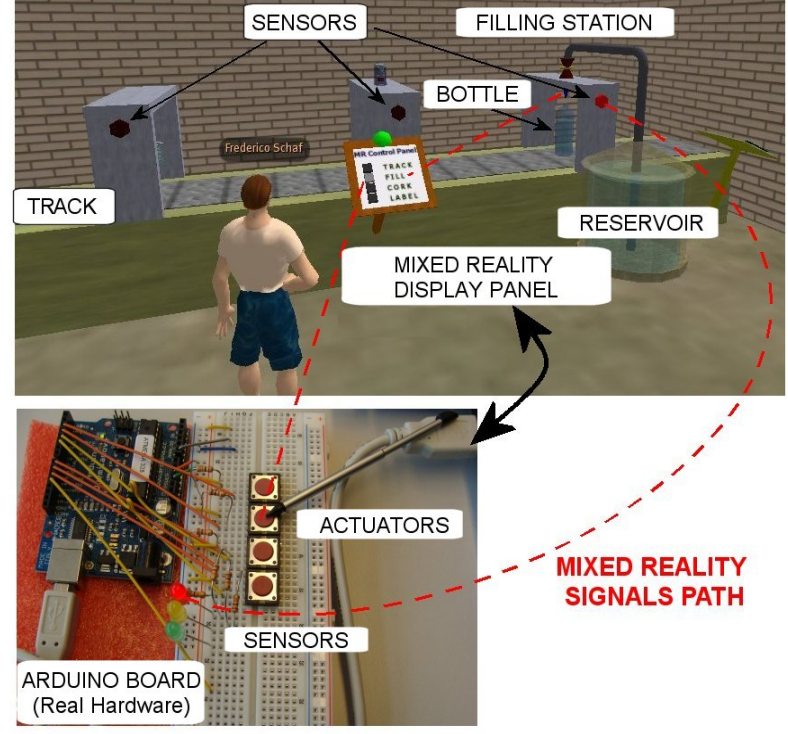

Figure 5. Hardware-in-the-Loop scheme with Arduino and the Virtual Bottling Plant

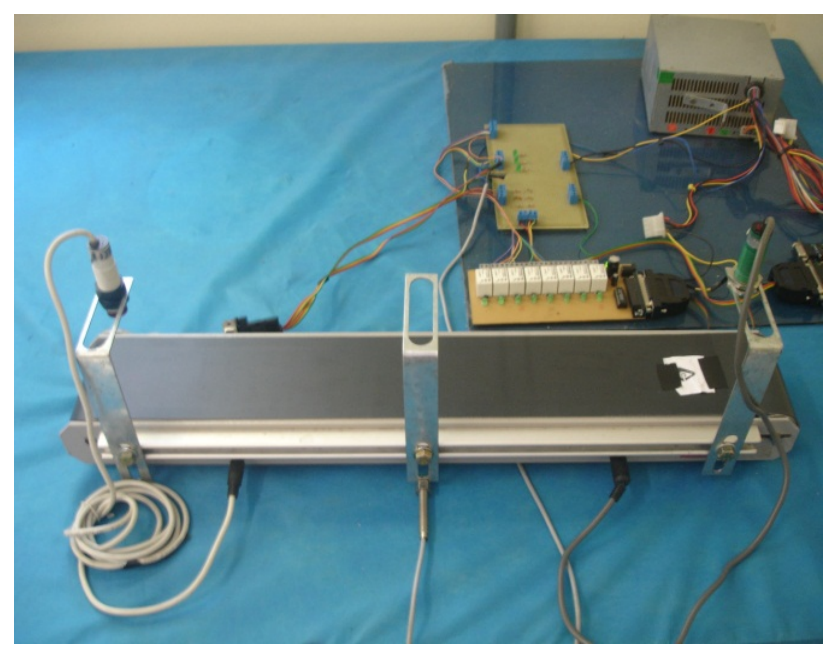

Figure 6. Simple bottling plant and hardware

on the MWS. A snapshot of this integration is depicted in the Figure 7 where real (combined to augmented reality) plant and virtual plant are synchronized.

One can argue, why should we use interchangeable components [14]? Considering the pros and cons of real (hand-on) vs. simulated experiments, one can see that in some sense they are complementary so that a combination of both becomes interesting. Simulations, although sometimes unrealistic, have some intrinsic characteristics that can be explored in different learning scenarios. One of the main advantages of using simulated labs is that they can be easily replicated. Students can use multiple copies (replicas) of the same simulation simultaneously. Another advantage of using simulation is that students can speed up slow dynamics systems for quick visualization. Safety concerns involving simulation variables limits are not as important as in real experiments since the simulated models cannot cause injuries.

This complementarity was the motivation to the creation of the interchangeable components strategy [14] that has been developed to allow the combination of both real and virtual components in control and automation educa- 
tion. The basic idea is that given that students usually have to use HMIs to access both real and simulated labs, and also due to the fact that usually these interfaces only provide visual and hearing feedback, they can be unable to differentiate if they are interacting with a real or a simulated device or technical plant. As described in [14], simulated components can be combined to real equipment to illustrate different learning situations (Hardware- or Software-in-the-Loop and totally real or totally simulated scenarios). For instance, a simulated plant can be used to evaluate possible effects of control actuation, avoiding damages to physical equipments that would occur in real plants in case of errors in the control algorithms. On the contrary, a simulated controller interacting with a real plant can be interesting in the sense that internal controller's variables and behavior can be better understood. The use of a simulated plant together with a simulated control algorithm can be very useful in activities such as parameter tuning, taking the already mentioned possibility offered by simulated systems, to speed up the behavior of slow technical processes allowing faster system identification.

\section{F. Collaboration during Immersive Learning}

The 3D AutoSysLab allows also collaboration during all immersive experience at the environment (see Figure 8). Each online user is represented by an avatar and the feeling of immersion is very present. Collaboration with in-world voice is also supported using the FreeSWITCH module from OpenSim.

Mixed reality interfaces allow the creation of shared workspaces that combine the advantages of both virtual environments and seamless collaboration with the real environment [46]. The information overlay is employed by remote collaborators to annotate the user's view, or may enhance face-to-face conversation producing shared interactive virtual models. In this way, mixed reality techniques can produce a shared sense of presence and reality [12]. Thus, mixed reality approaches are ideal for multi-user collaborative lab and work applications [46].

Due to its distributed nature, the 3D AutoSysLab allows the collaboration also in the practice (experiment) level. Users can also share their real hardware or simulation and connect it to the environment using XML-RPC servers. This way, they can develop their own controller/plant component and interconnect to the environment for testing, rapid prototyping or sharing of resources.

\section{G. Autonomous in-World Tutoring}

There is an ongoing study on automatic tutors modeled using the in LibOpenMetaverse library connected to JADE autonomous agents that collect and process information based on users interactions with the environment and between users. As this far only simple tutoring allied to experiments are modeled. This way, automated tutor avatars, so called tutor bots, were developed. This bots offer help and guidance each time a student comes close to a virtual experiment interface. The JADE multi-agent system collects experiment data stored on the MySQL database, and infers didactic materials or simple chat messages according to user's response to the guidance.

The Figure 9 illustrates how this approach is implemented in the 3D AutoSysLab when a student tries to use a virtual MPS pick-and-place module.

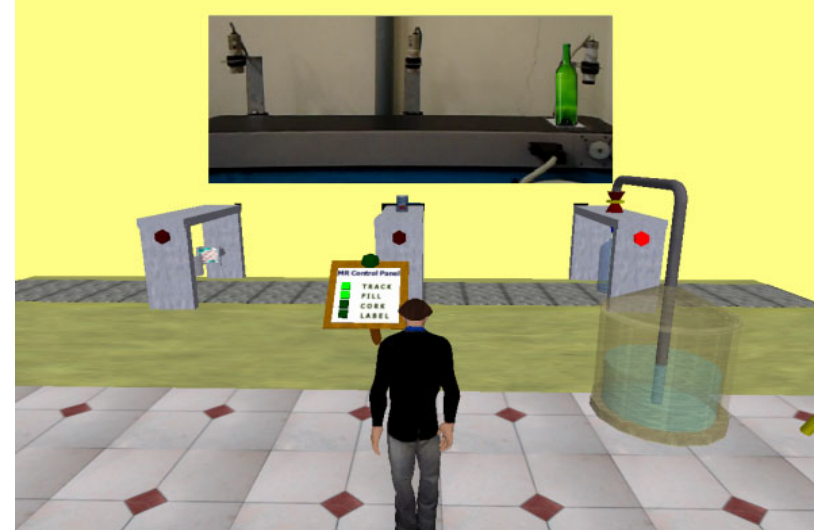

Figure 7. Real and virtual bottling plant integration
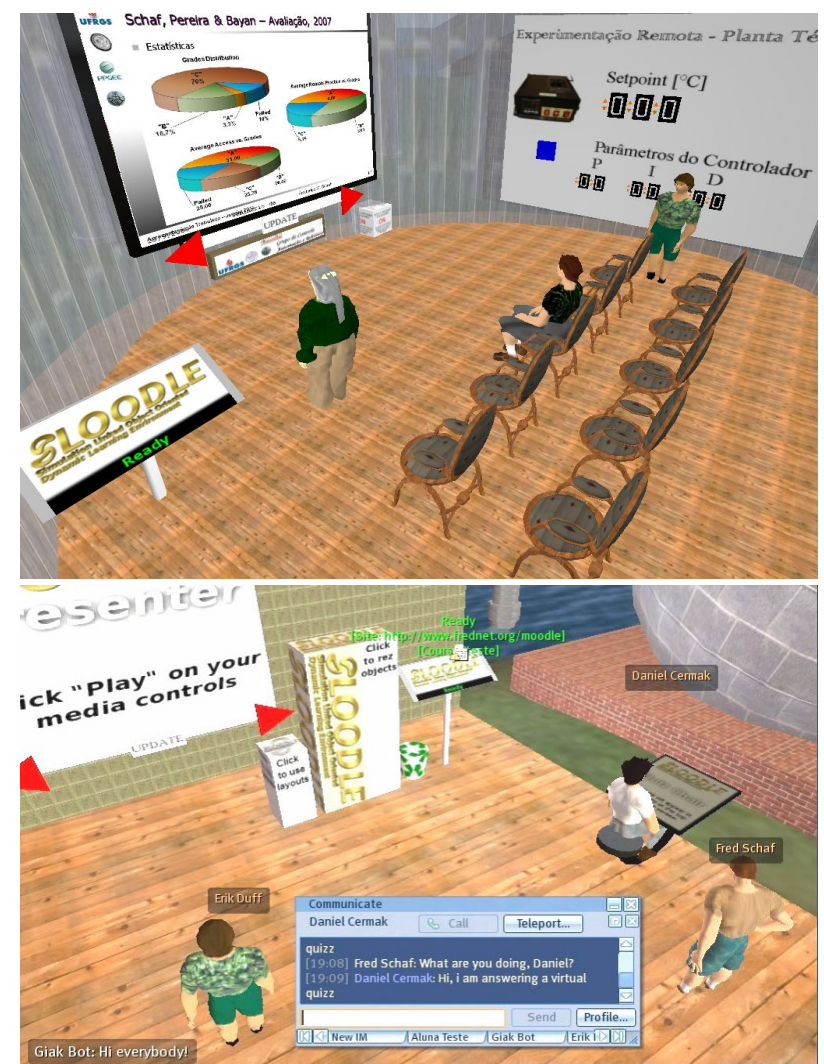

Figure 8. Collaboration in 3D AutoSysLab

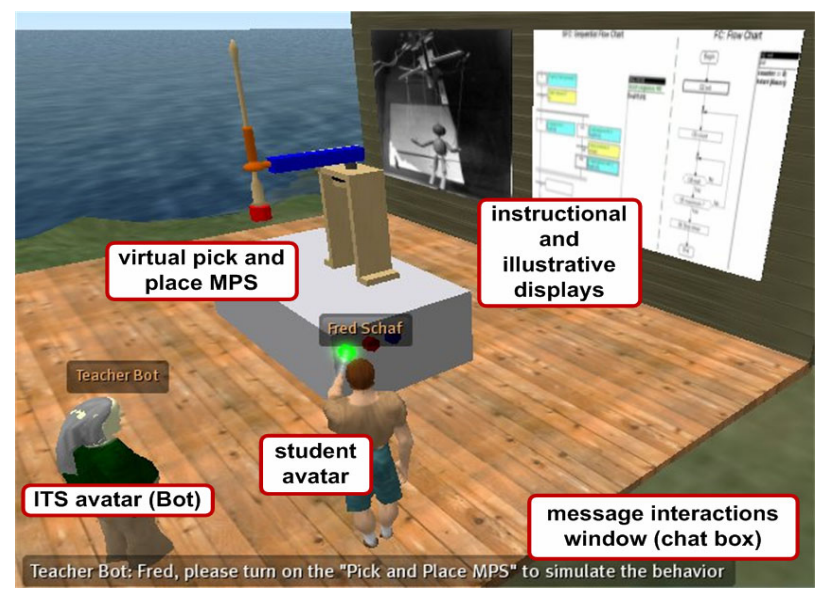

Figure 9. Tutor Bot in the metaverse simple guidance approach 


\section{CONCLUSIONS}

This work presented a research field that is the trend for future blended (distance) learning interfaces. Immersive environments can achieve higher level of user-user interactions, as well as user-system interaction by the feeling of immersion or the feeling of presence in the virtual world implementation.

Despite the early stages of usability the 3D AutoSysLab prototype motivates students, not only for its social perspective but also his game-like interface and the for its easy to use navigation and interface. The created metaverse is not limited the technical courses since metaverses can be created to several other research and educational areas.

This project is under development and will be tested next semester in several distinct courses, not only in control and automation systems, but also in English as Foreign Language (EFL) courses and digital inclusion courses.

The prototype software and hardware architecture can be increasingly enhanced since commonly, free and opensource resources were used to its development.

Until now only parts of the proposed system where tested but the results are very positive when applied to education of future engineers [44].

The evolution of e-learning systems in engineering education is evident when analyzing the history of handon, simulated, virtual, remote and interchangeable components labs. Our research group has covered all this implementations starting from a didactic plant for control systems [45] and conducted also an interesting evolutionary research presented in [43]. Regarding automation engineering education, an interesting aspect is that such CSCEs benefits from the same IT technologies and concepts that are being adopted in the development of modern automation systems and that have to be taught to students. So, by using these environments students are also getting acquainted with technologies they will find in real industrial automation systems. And this trend will probably continue, given that concepts such as cloud computing, self-adaptive/self-evolvable systems, whose application to control and automation systems are a current research topic, can also be adopted to improve educational environments.

A key issue to development of CSCE is the integration of different areas of knowledge, so that a multidisciplinary team required to plan and execute such project. This is very clear when analyzing the aspects of the use of technology (virtual environments, remote experiments, etc.) applied to education.

\section{FUTURE WORKS}

There are several other metaverses under development that will allow greater validation of the prototype as a larger audience of students will be using it.

Force-feedback interfaces, like haptic, as in [42] are being developed to be integrated in some experiments allowing the immersion of another senses. Most of the current implementations of virtual world labs, mixed reality, augmented reality and virtual reality labs rely only on computer mediated interfaces in their interactions with students. While those interfaces have an increasing capacity of generating very realistic visualizations, they usually only provide visual and hearing feedback to students, which can be enough for several applications, however, in some areas such as control and automation engineering education or medical training, other senses such as force, temperature, balance and acceleration are very important. This motivates future studies for the development of what can be called "multiple senses labs", which are virtual world labs with additional interfaces such as haptic, thermal, etc.

The integration of real intelligent tutoring system is also under analysis by the use of student models based not only in logged data but in a historic evolution of student learning status.

\section{ACKNOWLEDGMENT}

The authors thank all researchers that are directly or indirectly involved with the developed with the hardware as well as the developing community of the free and opensource softwares employed in this work.

\section{REFERENCES}

[1] B. Atkan; C. A. Bohus; L. A. Crowl and M. H. Shor, "Distance Learning Applied to Control Engineering Laboratories," IEEE Transactions On Education, vol. 39, pp. 320 - 326, Aug. 1996. http://dx.doi.org/10.1109/13.538754

[2] M. Cooper, "The Challenge of Practical Work in a eUniversity real, virtual and remote experiments", in: Proceedings of the Information Society Technologies Conference, 2000.

[3] T. Scheucher, P. H. Bailey, C. Gütl and V. J. Harward, “Collaborative Virtual 3D Environment for Internet-Accessible Physics Experiments,” International Journal of Online Engineering (iJOE), v.5, n.1, p.65-71, Aug. 2009.

[4] S. Poindexter and B. Heck, "Using the Web in your Courses: the How-To's and the Why's," American Control Conference, pp. 1304 - 1308, 1998.

[5] C. Schmid, "Virtual Control Laboratories and Remote Experimentation in Control Engineering," in Proceedings of the 11th EAEEIE Annual Conference on Innovations in Education for Electrical and Information Engineering (EIE), 2000.

[6] R. Marin, P. J. Sanz, P. Nebot and R. Wirz, "Multimodal interface to control a robot arm via the web: A case study on remote programming,” IEEE Transaction on Industrial Electronics, v. 52, (6), p. 1506-1520, Dec. 2005. http://dx.doi.org/10.1109/TIE.2005.858 $\underline{733}$

[7] L. Huijun and S. Aiguo, "Virtual-Environment Modeling and Correction for Force-Reflecting Teleoperation with Time Delay," IEEE Transactions on Industrial Electronics, v. 54 (2), p. $1227-$ 1233, Apr. 2007. http://dx.doi.org/10.1109/TIE.2007.893072

[8] T. P. Caudell et al., "Virtual Patient Simulator for Distributed Collaborative Medical Education," The Anatomical Record Part B: The New Anatomist, v. 270B (1), p. 23-29, Jan. 2003. http://dx.doi.org/10.1002/ar.b.10007

[9] F. M. Schaf and C. E. Pereira, “Automation and Control Learning Environment with Mixed Reality Remote Experiments Architecture,” International Journal of Online Engineering, vol. 3, 2007.

[10] G. Stahl, T. Koshmann and D. Suthers, "The Cambridge Handbook of the Learning Sciences,” Cambridge, UK: Cambridge University Press, p.209-452, 2006

[11] P. Milgram and F. Kishino, “A Taxonomy of Mixed-reality Visual Displays,” Transactions of the Institute of Electronics, Information and Communication Engineers on Information and Systems, v.E77-D (2), p.1321-1329, 1994.

[12] D. Müller, F. W. Bruns, H.-H. Erbe, B. Robben and Y.-H. Yoo, "Mixed Reality Learning Spaces for Collaborative Experimentation: a challenge for engineering education and training," International Journal of Online Engineering (iJOE), v. 3(4), 2007.

[13] N. Enlund, "Being Virtually There - Reality and Presence in Mediated Learning,” in: Proceedings of the 2001 International Conference on Telecommunications for Education and Training, v. 3(4), p. iv - ix, 2001. 
[14] F. M. Schaf and C. E. Pereira, "Integrating Mixed Reality Remote Experiments into Virtual Learning Environments using Interchangeable Components. IEEE Transactions on Industrial Electronics, v.56, p. 4776-4783, 2009. http://dx.doi.org/10.1109/TIE. 2009.2026369

[15] MOODLE Website, <http://www.moodle.org>

[16] M. Mc Mahon, "Social Constructivism and the World Wide Web A Paradigm for Learning," in: Proceedings of the Australian Society for Computers in Learning in Tertiary Education, 1997.

[17] Second Life Website, $<$ http://www.secondlife.com>

[18] Active Worlds Educational Universe, $<$ http://www.activeworlds.c om/edu $>$.

[19] Matlab, <http://www.mathworks.com>.

[20] LabView, <http://www.ni.com/labview $>$.

[21] M. Casini, D. Prattichizzo and A. Vicino, "The Automatic Control Telelab: a Web-based technology for distance learning," IEEE Control Systems Magazine, vol. 24, pp. 36 - 44, 2004. http://dx.doi.org/10.1109/MCS.2004.1299531

[22] I. Michaelides, P. Elefthreiou and D. Müller, "A Remotely Accessible Solar Energy Laboratory - A Distributed Learning Experience," in: Proceedings of the Remote Engineering and Virtual Instrumentation International Symposium (REV), Villach, Austria, 2004.

[23] R. T. Kouzes, J. D. Myers, and W. A. Wulf, "Collaboratories: Doing science on the Internet," IEEE Computer, 29(8), 40-46, 1996. http://dx.doi.org/10.1109/2.532044

[24] D. Müller, and J. M. M. Ferreira, "MARVEL: A mixed-reality learning environment for vocational training in mechatronics," in Proceedings of the Technology Enhanced Learning International Conference, p. 65-72, 2004.

[25] F. W. Bruns, "Hyper-Bonds - Distributed Collaboration in Mixed Reality," Annual Reviews in Control, v. 29 (1), p. 117-123, Mar. 2005. http://dx.doi.org/10.1016/j.arcontrol.2004.11.001

[26] F. M. Schaf, D. Müller, F. W. Bruns, C. E. Pereira and H. H. Erbe, "Collaborative Learning and Engineering Workspaces," Annual Reviews in Control, v. 33 (2), p. 246-252, Dec. 2009. http://dx.doi.org/10.1016/j.arcontrol.2009.05.002

[27] J. Kemp and S. J. Kabumpo, "Putting a Second Life 'Metaverse' Skin on Learning Management Systems," in Proceedings of the Second Life Education Workshop at Second Life Community Convention, San Francisco, USA, p.13-18, 2006.

[28] M. Callaghan, J. Harkin, T. M. McGinnity and L. P. Maguire, "Intelligent User Support in Autonomous Remote Experimentation Environments”, IEEE Transactions on Industrial Electronics, vol. 55(6), pp. 2355-2367, 2008. http://dx.doi.org/10.1109/TIE. 2008.922411

[29] MIT iLabs: Internet access to real labs - anywhere, anytime Website. $<$ http://icampus.mit.edu/ilabs $>$.

[30] Open Wonderland Website, <http://openwonderland.org>.

[31] A. Sliney, D. Murphy, "JDoc: A Serious Game for Medical Learning," in: Proceedings of the First International Conference on Advances in Computer-Human Interaction, pp.131-136, 2008. http://dx.doi.org/10.1109/ACHI.2008.50

[32] F M. Schaf, “Arquitetura Modular para Ambientes Virtuais de Ensino de Automação com Suporte à Realidade Mista e
Colaboração,” PhD Thesis available only in Portuguese: $<$ http://hdl.handle.net/10183/10320>.

[33] Open Simulator Website, <http://opensimulator.org>.

[34] Hippo OpenSim Viewer Website, <http://mjm-labs.com/viewer >.

[35] SLOODLE Project Website, <http://www.sloodle.org >.

[36] JADE Java Agent DEvelopment Framework Website, $<$ http://jade.tilab.com>.

[37] MySQL Website, <http://www.mysql.com>.

[38] LibOpenMetarverse Website, <http://openmetaverse.org/projects/ libopenmetaverse $>$

[39] Arduino Website, <http://www.arduino.cc $>$.

[40] Microservidor Web, $<$ http://www.rexlab.net $>$.

[41] ARToolKit Website, <http://www.hitl.washington.edu/artoolkit>.

[42] Y. H. Yoo and F. W. Bruns, "Realtime Collaborative Mixed Reality Environment with Force Feedback," in Proceedings of the 7th IFAC Symposium on Cost Oriented Automation, Ottawa, 2004.

[43] C. E. Pereira, F. M. Schaf and S. Paladini, "Control and Automation Engineering Education: combining physical, remote and virtual labs," in Proceedings of the 9th International MultiConference on Systems, Signals and Devices (SSD), Chemnitz, Germany, 2012.

[44] F. M. Schaf, C. E. Pereira and D. Müller, "Collaborative Environment Architecture Proposal: A study of virtual environments for learning purposes in control engineering," in Proceedings of the International Conference on Remote Engineering and Virtual Instrumentation (REV), Stockholm, 2010.

[45] R. P. Zeilmann, J. M. G da Silva, A. S. Bazanella and C. E. Pereira, "Web-based Control Experiment on a Foundation Fieldbus Pilot Plant," in Proceedings of the IFAC International Conference on Fieldbus Systems and their Applications, Aveiro, Portugal, pp. 325 - 330, 2003.

[46] F. M. Schaf et al., "Collaborative Learning Environment using Distributed Mixed Reality Experiment for Teaching Mechatronics," in Proceedings of the 8th IFAC Symposium on Cost Oriented Automation, Habana, Cuba, 2007.

\section{AUTHORS}

F. M. Schaf is a professor at the Educational Technology Center from the Universidade Federal de Santa Maria, Santa Maria, RS, 97105-900, Brazil (e-mail: frederico.schaf@nte.ufsm.br).

S. Paladini is a $\mathrm{PhD}$ candidate at Universidade Federal do Rio Grande do Sul, Porto Alegre, RS, Brazil (e-mail: suenoni.paladini@ufrgs.br).

C. E. Pereira is a professor at the Electrical Engineering Department from the Universidade Federal do Rio Grande do Sul, Porto Alegre, RS, Brazil (e-mail: cpereira@ece.ufrgs.br).

This article is an extended version of a paper presented at the International Conference IEEE EDUCON2012, held in April 2012, in Marrakesh, Morocco. Received, 22 April 2012. Published as resubmitted by the authors 30 April 2012. 